\title{
Electric-Magnetic Duality in QED Effective Action
}

\author{
W. S. Bae and Y. M. Cho \\ Department of Physics, College of Natural Sciences, Seoul National University, Seoul 151-742, Korea \\ hypersym@zoo.snu.ac.kr, ymcho@yongmin.snu.ac.kr \\ and \\ D. G. Pak \\ Asia Pacific Center for Theoretical Physics, 207-43 Cheongryangri-dong, Dongdaemun-gu, Seoul 130-012 Korea \\ dmipak@mail.apctp.org
}

\begin{abstract}
Recently we have obtained a non-perturbative but convergent series expression of the one loop effective action of QED, and discussed the renormalization of the effective action. In this paper we establish the electric-magnetic duality in the quantum effective action.
\end{abstract}

PACS numbers: 12.20.-m, 13.40.-f, 11.10.Jj, 11.15.Tk

The effective theory of QED plays a crucial role in our understanding of the non-linear effects in electrodynamics. Recently we have obtained a convergent series expression of the effective action of QED in one loop approximation, and established the renormalization group invariance of the effective action [1]. A remarkable feature of the effective action is the electric-magnetic duality,a fundamental symmetry of the quantum effective action of QED. The purpose of this paper is to provide a concrete proof of the duality in the effective action of QED.

The effective action of QED has been studied by Euler and Heisenberg and by Schwinger long time ago [2,3], and by many others later [4,5].To derive the effective action one may start from the QED Lagrangian

$$
\begin{gathered}
\mathcal{L}=-\frac{1}{4} F_{\mu \nu}^{2}+\bar{\Psi}(i \not D-m) \Psi, \\
D_{\mu}=\partial_{\mu}+i e A_{\mu},
\end{gathered}
$$

where $m$ is the electron mass. With a proper gauge fixing one can show that the electron one loop correction of the effective action is given by

$$
\Delta S=i \ln \operatorname{Det}(i \not D-m) .
$$

So for an arbitrary constant background one has [2, 3]

$$
\Delta \mathcal{L}=-\frac{a b}{8 \pi^{2}} \int_{0+i \epsilon}^{\infty+i \epsilon} \frac{d t}{t} \operatorname{coth}(a t) \cot (b t) \exp \left(-m^{2} t\right)
$$

where

$$
\begin{aligned}
a & =\frac{e}{2} \sqrt{\sqrt{F^{4}+(F \tilde{F})^{2}}+F^{2}}, \\
b & =\frac{e}{2} \sqrt{\sqrt{F^{4}+(F \tilde{F})^{2}}-F^{2}} .
\end{aligned}
$$

Notice that the above contour of the integral is dictated by the causality. Although the integral expression (3) looks innocent, it has been non-trivial to perform the integral for an arbitrary constant background [4, 5 . But we can integrate this and obtain a convergent series expression of the effective action with the help of the Sitaramachandrarao's identity [6],

$$
\begin{gathered}
x y \operatorname{coth} x \cot y=1+\frac{1}{3}\left(x^{2}-y^{2}\right) \\
-\frac{2}{\pi} x^{3} y \sum_{n=1}^{\infty} \frac{1}{n} \frac{\operatorname{coth}\left(\frac{n \pi y}{x}\right)}{\left(x^{2}+n^{2} \pi^{2}\right)} \\
+\frac{2}{\pi} x y^{3} \sum_{n=1}^{\infty} \frac{1}{n} \frac{\operatorname{coth}\left(\frac{n \pi x}{y}\right)}{\left(y^{2}-n^{2} \pi^{2}\right)} .
\end{gathered}
$$

A preliminary version of the identity which was expressed as a divergent asymptotic series was obtained by Ramanujan, and Sitaramachandrarao improved the identity in the above convergent series expression. To carry out the integral (3)and express it as a convergent series expression we need the Sitaramachandrarao's identity. Indeed with the identity we obtain the following one loop effective action for an arbitrary constant background (after the modified minimal subtraction) [1:] ]

$$
\begin{aligned}
& \mathcal{L}_{\text {eff }}=-\frac{a^{2}-b^{2}}{2 e^{2}}\left(1-\frac{e^{2}}{12 \pi^{2}} \ln \frac{m^{2}}{\mu^{2}}\right) \\
& -\frac{a b}{4 \pi^{3}} \sum_{n=1}^{\infty} \frac{1}{n}\left[\operatorname { c o t h } ( \frac { n \pi b } { a } ) \left(\operatorname{ci}\left(\frac{n \pi m^{2}}{a}\right) \cos \left(\frac{n \pi m^{2}}{a}\right)\right.\right. \\
& \left.+\operatorname{si}\left(\frac{n \pi m^{2}}{a}\right) \sin \left(\frac{n \pi m^{2}}{a}\right)\right) \\
& -\frac{1}{2} \operatorname{coth}\left(\frac{n \pi a}{b}\right)\left(\exp \left(\frac{n \pi m^{2}}{b}\right) \operatorname{Ei}\left(-\frac{n \pi m^{2}}{b}\right)\right.
\end{aligned}
$$




$$
\left.\left.+\exp \left(-\frac{n \pi m^{2}}{b}\right) \operatorname{Ei}\left(\frac{n \pi m^{2}}{b}-i \epsilon\right)\right)\right]
$$

where $\mu$ is the subtraction parameter, and $\operatorname{ci}(x), \operatorname{si}(x)$, and $\operatorname{Ei}(-x)$ are the cosine, sine, and exponential integral functions given by (for $\operatorname{Re} x>0$ ) [8],

$$
\begin{aligned}
\operatorname{ci}(x) & =-\int_{x}^{\infty} \frac{\cos (t)}{t} d t \\
& =\gamma+\ln x+\sum_{n=1}^{\infty} \frac{(-1)^{n} x^{2 n}}{(2 n) ! 2 n}, \\
\operatorname{si}(x) & =-\int_{x}^{\infty} \frac{\sin (t)}{t} d t \\
& =-\frac{\pi}{2}-\sum_{n=1}^{\infty} \frac{(-1)^{n} x^{2 n-1}}{(2 n-1) !(2 n-1)} \\
\operatorname{Ei}(-x) & =-\int_{x}^{\infty} \frac{e^{-t}}{t} d t \\
& =\gamma+\ln x+\sum_{n=1}^{\infty} \frac{(-1)^{n} x^{n}}{(n) ! n} .
\end{aligned}
$$

Notice that our series expression of the effective action is non-perturbative but convergent.

The effective action (5) nicely reproduces the wellknown imaginary part when $b \neq 0$ [泡],

$$
\operatorname{Im} \mathcal{L}_{\text {eff }}=\frac{a b}{8 \pi^{2}} \sum_{n=1}^{\infty} \frac{1}{n} \operatorname{coth}\left(\frac{n \pi a}{b}\right) \exp \left(-\frac{n \pi m^{2}}{b}\right) \text {. }
$$

This is because the exponential integral function $\operatorname{Ei}(-x)$ in (5) develops an imaginary part $i \pi$ after the analytic continuation from $-x$ to $x[1]$. The important point here is that one should make the analytic continuation in such a way to preserve the causality, which determines the signature of the imaginary part in (7). The physical meaning of the imaginary part is well known [3]. The electric background generates the pair creation, with the probability per unit volume per unit time given by (7).

Another important aspect of the effective action is the logarithmic correction $\ln (m / \mu)^{2}$ of the classical part of the action. This indicates that even the classical part of the action gets the quantum correction. One might think that this correction is unphysical and should disappear after the renormalization, since one can remove this logarithmic correction by choosing $\mu=m$. But this is not true. Indeed it has been shown that there exists a real physical quantum correction to the classical part of the action even after the renormalization [1].

Notice that in the pure magnetic and the pure electric background the effective action (5) reduces to

$$
\mathcal{L}_{e f f}=-\frac{a^{2}}{2 e^{2}}\left(1-\frac{e^{2}}{12 \pi^{2}} \ln \frac{m^{2}}{\mu^{2}}\right)
$$

$$
\begin{aligned}
& -\frac{a^{2}}{4 \pi^{4}} \sum_{n=1}^{\infty} \frac{1}{n^{2}}\left(\operatorname{ci}\left(\frac{n \pi m^{2}}{a}\right) \cos \left(\frac{n \pi m^{2}}{a}\right)\right. \\
& \left.+\operatorname{si}\left(\frac{n \pi m^{2}}{a}\right) \sin \left(\frac{n \pi m^{2}}{a}\right)\right)
\end{aligned}
$$

and to

$$
\begin{gathered}
\mathcal{L}_{\text {eff }}=\frac{b^{2}}{2 e^{2}}\left(1-\frac{e^{2}}{12 \pi^{2}} \ln \frac{m^{2}}{\mu^{2}}\right) \\
+\frac{b^{2}}{8 \pi^{4}} \sum_{n=1}^{\infty} \frac{1}{n^{2}}\left(\exp \left(\frac{n \pi m^{2}}{b}\right) \operatorname{Ei}\left(-\frac{n \pi m^{2}}{b}\right)\right. \\
\left.+\exp \left(-\frac{n \pi m^{2}}{b}\right) \overline{\operatorname{Ei}}\left(\frac{n \pi m^{2}}{b}\right)\right) \\
+i \frac{b^{2}}{8 \pi^{3}} \sum_{n=1}^{\infty} \frac{1}{n^{2}} \exp \left(-\frac{n \pi m^{2}}{b}\right)
\end{gathered}
$$

where

$$
\begin{aligned}
\overline{\operatorname{Ei}}(x) & =\frac{1}{2}(\operatorname{Ei}(x+i \epsilon)+\operatorname{Ei}(x-i \epsilon)) \\
& =\operatorname{Re} \operatorname{Ei}(x) . \quad(x>0)
\end{aligned}
$$

Although the above effective actions for the pure electric and the pure magnetic backgrounds look totally different, they are actually very closely related. In fact one can show that they are the mirror image of each other, so that one can obtain one from the other simply by making a dual transformation [1]. This is a first indication that the quantum effective action has a fundamental symmetry which we call the duality.In general the duality asserts that the effective action of QED is manifestly invariant under the dual transformation,

$$
a \rightarrow-i b, \quad b \rightarrow i a
$$

This means that the effective action, as a function of $z=a+i b$, is invariant under the reflection from $z$ to $-z$. Notice that, in the Lorentz frame where $\vec{E}$ is parallel to $\vec{B}, a$ becomes $B$ and $b$ becomes $E$. So the duality describes the electric-magnetic duality.

One might think that the duality is obvious since it immediately follows from the integral expression (3). But we emphasize that this is not so. In fact the integral expression is invariant under the four different transformations,

$$
a \rightarrow \pm i b, \quad b \rightarrow \pm / \mp i a .
$$

But among the four only our duality (11) survives as the true symmetry of the effective action. This tells that the duality constitutes a non-trivial symmetry of the quantum effective action.

To establish the duality it is important to realize that the dual transformation automatically involves the analytic continuation of the special functions $\operatorname{ci}(x), \operatorname{si}(x)$, 
and $\operatorname{Ei}(-x)$ in (5).So one must find the correct analytic continuation to establish the duality in the effective action.To do this we observe from (6) that [8]

$$
\begin{aligned}
& \operatorname{ci}(x)=\frac{1}{2}(\operatorname{Ei}(i x)+\operatorname{Ei}(-i x)) \\
& \operatorname{si}(x)=\frac{1}{2 i}(\operatorname{Ei}(i x)-\operatorname{Ei}(-i x)) .
\end{aligned}
$$

Notice that although $\operatorname{ci}(x)$ and $\operatorname{si}(x)$ look like an even and odd function respectively, they are not. This is because $\operatorname{Ei}(x)$ has the branch cut along the positive real axis. From (13) we have for a positive real $x$

$$
\begin{gathered}
\operatorname{ci}( \pm i x)=\frac{1}{2}(\operatorname{Ei}(-x)+\overline{\operatorname{Ei}}(x)) \pm i \frac{\pi}{2} \\
\operatorname{si}( \pm i x)= \pm \frac{1}{2 i}(\operatorname{Ei}(-x)-\overline{\operatorname{Ei}}(x))-\frac{\pi}{2},
\end{gathered}
$$

and

$$
\operatorname{Ei}( \pm i x)=\operatorname{ci}(x) \pm i \operatorname{si}(x)
$$

Furthermore from (10) and (13) we have (for a positive real $x$ )

$$
\begin{aligned}
\overline{\operatorname{Ei}}(i x) & =\frac{1}{2}\left(\operatorname{Ei}(i x)+\operatorname{Ei}^{(+)}(i x)\right) \\
& =\operatorname{Ei}(i x)+i \pi \\
\overline{\operatorname{Ei}}(-i x) & =\frac{1}{2}\left(\operatorname{Ei}^{(-)}(-i x)+\operatorname{Ei}(-i x)\right) \\
& =\operatorname{Ei}(-i x)-i \pi,
\end{aligned}
$$

where $\mathrm{Ei}^{( \pm)}( \pm i x)$ are $\operatorname{Ei}( \pm i x)$ on the $( \pm 1)$-th Riemann sheets.

With the above preliminaries we can now establish the duality. To do this let us express the effective action (5) as

$$
\begin{gathered}
\mathcal{L}_{e f f}=-\frac{a^{2}-b^{2}}{2 e^{2}}\left(1-\frac{e^{2}}{12 \pi^{2}} \ln \frac{m^{2}}{\mu^{2}}\right) \\
-\frac{a b}{4 \pi^{3}} \sum_{n=1}^{\infty} \frac{1}{n}\left[\operatorname{coth}\left(\frac{n \pi b}{a}\right) f_{1}(n x)\right. \\
\left.-\operatorname{coth}\left(\frac{n \pi a}{b}\right) f_{2}(n y)\right]
\end{gathered}
$$

where

$$
\begin{gathered}
f_{1}(n x)=\operatorname{ci}(n x) \cos (n x)+\operatorname{si}(n x) \sin (n x), \\
f_{2}(n y)=\frac{1}{2}(\operatorname{Ei}(-n y) \exp (n y) \\
+(\overline{\operatorname{Ei}}(n y)+i \pi) \exp (-n y)), \\
x=\frac{\pi m^{2}}{a}, \quad y=\frac{\pi m^{2}}{b} .
\end{gathered}
$$

Now under

$$
a \rightarrow \mp i b \quad \text { or } \quad x \rightarrow \pm i y
$$

we have from (14)

$$
\begin{gathered}
f_{1}(n x) \rightarrow\left(\frac{1}{2}(\operatorname{Ei}(-n y)+\overline{\operatorname{Ei}}(n y)) \pm i \frac{\pi}{2}\right) \cosh (n y) \\
+\left(\frac{1}{2}(\operatorname{Ei}(-n y)-\overline{\operatorname{Ei}}(n y)) \mp i \frac{\pi}{2}\right) \sinh (n y) \\
=\frac{1}{2}(\operatorname{Ei}(-n y) \exp (n y) \\
+(\overline{\operatorname{Ei}}(n y) \pm i \pi) \exp (-n y))
\end{gathered}
$$

From this we have

$$
\begin{gathered}
f_{1}(n x) \rightarrow f_{2}(n y) \text { for } a \rightarrow-i b, \\
f_{1}(n x) \rightarrow f_{2}(n y)-i \pi \exp (-n y) \text { for } a \rightarrow+i b .
\end{gathered}
$$

On the other hand under

$$
b \rightarrow \pm i a \quad \text { or } \quad y \rightarrow \mp i x
$$

we have from (15) and (16)

$$
\begin{gathered}
f_{2}(n y) \rightarrow \frac{1}{2}(\operatorname{ci}(n x) \pm i \operatorname{si}(n x)) \\
(\cos (n x) \mp i \sin (n x)) \\
+\frac{1}{2}(\operatorname{ci}(x) \mp i \operatorname{si}(x) \mp i \pi+i \pi) \\
(\cos (n x) \pm i \sin (n x)) \\
=\left(\operatorname{ci}(n x)+\frac{i}{2}(\pi \mp \pi)\right) \cos (n x) \\
+\left(\operatorname{si}(n x)+\frac{1}{2}(\pi \mp \pi)\right) \sin (n x) .
\end{gathered}
$$

From this we have

$$
\begin{gathered}
f_{2}(n y) \rightarrow f_{1}(n x) \text { for } b \rightarrow+i a \\
f_{2}(n y) \rightarrow f_{1}(n x)+i \pi \exp (-i n x) \text { for } b \rightarrow-i a .
\end{gathered}
$$

From these it must become clear that the effective action is invariant under the dual transformation (11). As importantly the above analysis shows that the effective action is not invariant under the other three transformations of (12). This establishes the duality in the quantum effective action in QED.It is really remarkable that the quantum effective action is invariant only under our duality (11), even though the integral expression (3) appears to be invariant under the four different transformations (12).

One can obtain the similar results for the scalar QED. In this case the integral expression of the one loop correction is given by [3, 4 . 


$$
\Delta \mathcal{L}_{0}=\frac{a b}{16 \pi^{2}} \int_{0+i \epsilon}^{\infty+i \epsilon} \frac{d t}{t} \operatorname{csch}(a t) \csc (b t) \exp \left(-m^{2} t\right)
$$

To perform the integral we need a new identity similar to the Sitaramachandrarao's identity (4)

$$
\begin{gathered}
x y \operatorname{csch} x \csc y=1-\frac{1}{6}\left(x^{2}-y^{2}\right) \\
-\frac{2}{\pi} x^{3} y \sum_{n=1}^{\infty} \frac{(-1)^{n}}{n} \frac{\operatorname{csch}\left(\frac{n \pi y}{x}\right)}{x^{2}+n^{2} \pi^{2}} \\
+\frac{2}{\pi} x y^{3} \sum_{n=1}^{\infty} \frac{(-1)^{n}}{n} \frac{\operatorname{csch}\left(\frac{n \pi x}{y}\right)}{y^{2}-n^{2} \pi^{2}} .
\end{gathered}
$$

Again a preliminary version which was divergent was obtained by Ramanujan [6], but we have improved the Ramanujan's identity and obtained the above convergent series [1]. With this identity we can integrate (24) and express the effective action as (with the modified minimal subtraction)

$$
\begin{gathered}
\mathcal{L}_{0 \text { eff }}=-\frac{a^{2}-b^{2}}{2 e^{2}}\left(1-\frac{e^{2}}{48 \pi^{2}} \ln \frac{m^{2}}{\mu^{2}}\right) \\
+\frac{a b}{8 \pi^{3}} \sum_{n=1}^{\infty} \frac{(-1)^{n}}{n}\left[\operatorname{csch}\left(\frac{n \pi b}{a}\right) f_{1}(n x)\right. \\
\left.-\operatorname{csch}\left(\frac{n \pi a}{b}\right) f_{2}(n y)\right] .
\end{gathered}
$$

With this it is now straightforward to establish the duality in the effective action of the scalar QED. Indeed from (20) and (23) it must be clear that the effective action is invariant under the dual transformation (11). Notice again that the integral expression of the effective action (24) is invariant under the four different transformations (12), but the quantum effective action (26) is invariant under only our duality (11).

In the pure magnetic background the one loop effective action (26) reduces to [1]

$$
\begin{gathered}
\mathcal{L}_{0 \text { eff }}=-\frac{a^{2}}{2 e^{2}}\left(1-\frac{e^{2}}{48 \pi^{2}} \ln \frac{m^{2}}{\mu^{2}}\right) \\
+\frac{a^{2}}{8 \pi^{4}} \sum_{n=1}^{\infty} \frac{(-1)^{n}}{n^{2}}\left(\operatorname{ci}\left(\frac{n \pi m^{2}}{a}\right) \cos \left(\frac{n \pi m^{2}}{a}\right)\right. \\
\left.+\operatorname{si}\left(\frac{n \pi m^{2}}{a}\right) \sin \left(\frac{n \pi m^{2}}{a}\right)\right),
\end{gathered}
$$

and to

$$
\begin{gathered}
\mathcal{L}_{0 \text { eff }}=\frac{b^{2}}{2 e^{2}}\left(1-\frac{e^{2}}{48 \pi^{2}} \ln \frac{m^{2}}{\mu^{2}}\right) \\
-\frac{b^{2}}{16 \pi^{4}} \sum_{n=1}^{\infty} \frac{(-1)^{n}}{n^{2}}\left(\exp \left(\frac{n \pi m^{2}}{b}\right) \operatorname{Ei}\left(-\frac{n \pi m^{2}}{b}\right)\right.
\end{gathered}
$$

$$
\begin{gathered}
\left.+\exp \left(-\frac{n \pi m^{2}}{b}\right) \overline{\operatorname{Ei}}\left(\frac{n \pi m^{2}}{b}\right)\right) \\
-i \frac{b^{2}}{16 \pi^{3}} \sum_{n=1}^{\infty} \frac{(-1)^{n}}{n^{2}} \exp \left(-\frac{n \pi m^{2}}{b}\right) .
\end{gathered}
$$

Now, it is clear that we can obtain one from the other by the dual transformation (11). So one need to know only the effective action for the pure magnetic background to know the effective action for the pure electric background, and vise versa. This demonstrates the power of the duality.

In this paper we have established the electricmagnetic duality in the quantum effective action of the standard QED and the scalar QED. From the physical point of view the existence of the duality in the effective action is perhaps not so surprising. But the fact that this duality is borne out from our calculation of one loop effective action is really remarkable. We expect the duality to bean exact symmetry of the quantum effective action of gauge theories for an arbitrary $n$-loop approximation.

One can show that exactly the same duality exists in non-Abelian gauge theories, in particular in QCD [9]. So it must be clear that the duality is a fundamental symmetry of the quantum effective action of the gauge theories, both Abelian and non-Abelian. This means that we can use the duality as a consistency check of the correctness of the quantum effective action of gauge theories.A more detailed discussion on the subject will be published elsewhere [10].

One of the authors (YMC) thanks Professor C. N. Yang for the illuminating discussions. The work is supported in part by Korea Research Foundation (KRF2000-015-BP0072), and by the BK21 project of Ministry of Education.

[1] Y. M. Cho and D. G. Pak, hep-th/0006057, hepth/0010073.

[2] W. Heisenberg and H. Euler, Z. Phys. 98 (1936) 714; V. Weisskopf, Kgl. Danske Vid. Sel. Mat. Fys. Medd. 14, 6 (1936).

[3] J. Schwinger, Phys. Rev. 82 , 664 (1951); Phys. Rev. 128, 2425 (1962).

[4] A. Nikishov, JETP 30, 660 (1970); W. Dittrich, J. Phys. A9, 1171 (1976); J. Dowker and R. Critchley, Phys. Rev. D13, 3224 (1976); M. Claudson, A. Yilditz, and P. Cox, Phys. Rev. D22, 2022 (1980); S. Blau, M. Visser, and A. Wipf, Int. J. Mod. Phys.A6, 5409 (1991); J. Heyl and L. Hernquist, Phys. Rev. D55, 2449 (1997); R. Soldati and L. Sorbo, Phys. Lett. B426, 82 (1998); G. Dunne and T. Hall, Phys. Rev. D60, 065002 (1999); C. Beneventano and E. Santangelo, hep-th/0006123. 
[5] V. Ritus, JETP 42, 774 (1976); 46, 423 (1977); M. Reuter, M. Schmidt, and C. Schubert, Ann. Phys, 259, 313 (1997).

[6] R. Sitaramachandrarao, in Ramanujan's Notebooks Vol. 2, p271, edited by B. C. Berndt (Springer-Verlag) 1989.

[7] W. Mielniczuk, J. Phys. A15, 2905 (1982). It has been asserted by Jentschura and Weniger in hep-th/0007108 that our expression (5) of the effective action has already appeared in this paper. This assertion, however, is not based on the facts. Indeed this paper is based on the incorrect identity with which one can not possibly obtain the desired result. See D. G. Pak, hep-th/0010316, for more details.

[8] I. Gradshteyn and I. Ryzhik, Table of Integrals, Series, and Products, edited by A. Jeffery (Academic Press) 1994; M. Abramowitz and I. Stegun, Handbook of Mathematical Functions, (Dover) 1970.

[9] Y. M. Cho and D. G. Pak, hep-th/0006051, submitted to Phys. Rev. D.

[10] W. S. Bae, Y. M. Cho, and D. G. Pak, to be published. 